Check for updates

Cite this: Mater. Chem. Front., 2017, 1, 1995

Received 20th January 2017, Accepted 6th June 2017

DOI: $10.1039 / \mathrm{c} 7 \mathrm{qm} 00028 \mathrm{f}$

rsc.li/frontiers-materials

\section{In situ polymerized hyperbranched polymer reinforced poly(acrylic acid) hydrogels}

\author{
Nazila Dehbari, $\uparrow^{\mathrm{a}}$ Javad Tavakoli, $\dagger^{\mathrm{b}}$ Simranjeet Singh Khatrao ${ }^{\mathrm{ab}}$ and \\ Youhong Tang id *a
}

\begin{abstract}
Hydrogels have been extensively investigated for use in various applications. Poly acrylic acid (PAA) is a common example, which has been widely used due to its super hydrophilicity properties, biocompatibility and biodegradability characteristics. However its poor mechanical properties, which have been addressed in many research studies, are known as a drawback that limits its applications. So, enhancing PAA mechanical properties using a hyperbranched polymer $(\mathrm{HB})$ is the key question to be addressed in this research. Investigations of the mechanical properties of the PAA-HB hydrogel revealed $130 \%$ improvement in the ultimate tensile strength, indicating a two times enhancement compared to that of PAA. Statistical analysis showed that the overall effect of introducing notches (with different depths) on the selected mechanical properties of both PAA and PAA-HB was significant. Mechanical characterization of PAA-HB networks showed that significant improvement in the mechanical properties was achieved as the capability of water uptake increased by $20 \%$. Characterization of the physical properties confirmed that participation of HB may form a PAA based hybrid material with good swelling properties. Those findings are attributed to the supramolecular structure of the HB, which can introduce physical entanglement between the PAA network structure and increase the crystallinity of the final hydrogel as compared to those from the PAA hydrogel.
\end{abstract}

\section{Introduction}

Hydrogels are three-dimensional cross-linked, water-swollen and hydrophilic polymers whose properties are highly dependent on the environmental conditions. ${ }^{1-3}$ As enviro-sensitive materials, hydrogels have been widely used for industrial and biomedical applications due to their unique structure and capabilities. ${ }^{4-6}$ The properties of a specific hydrogel are extremely important in selecting which materials are suitable for a given application. The suitability of a hydrogel for a given application depends on the mechanical properties that are affected by the environmental conditions such as temperature, $\mathrm{pH}$ level and ionic strength, magnetic and electric fields as well as the monomer type and crosslinking agents. $^{7-9}$

Among various types of hydrogels, poly acrylate polymers such as poly acrylic acid (PAA), with distinctive absorptivity properties, have the most versatile structure to enhance their practical utility in everyday life applications such as filtration, water remediation, diapers and hygiene products, cosmetics,

\footnotetext{
${ }^{a}$ Centre for NanoScale Science and Technology, School of Computer Science, Engineering and Mathematics, Flinders University, South Australia 5042, Australia. E-mail: youhong.tang@flinders.edu.au; Tel: +61-8-82012138

${ }^{b}$ The Medical Device Research Institute (MDRI), School of Computer Science, Engineering and Mathematics, Flinders University, South Australia 5042, Australia $\dagger$ These authors contributed equally.
}

wound dressings, medical waste solidification and metal ion removal. ${ }^{10-14}$ From extensive studies evaluating different properties of PAA hydrogels, such as swelling, ${ }^{15}$ adhesion, ${ }^{16}$ diffusion, ${ }^{17}$ physico-chemical $^{18}$ and mechanical ${ }^{19}$ properties, it has been revealed that the greatest concern about these types of hydrogels is their poor mechanical properties. ${ }^{11,20-22}$ The poor mechanical properties of PAA hydrogels, which relate closely to their large volume change subsequent to swelling, count as a marked weakness in consideration for adoption for high-tech applications, and have limited their specific applications. ${ }^{23,24}$ Typically, PAA hydrogels exhibit a low Young's modulus $(<200 \mathrm{kPa})$ and low fracture energy $\left(<20 \mathrm{~J} \mathrm{~m}^{-2}\right)$, and possess a low tensile strength $(<0.2 \mathrm{MPa}) .^{25,26}$ Therefore, there has been an urgent focus on improving the mechanical properties of PAA hydrogels via toughening methods by mixing different types of crosslinked polymers or introducing energy-dissipating mechanisms. ${ }^{27-29}$ Various methods of producing multi-functional cross-linked hydrogels, ${ }^{30}$ nanocomposite hydrogels, ${ }^{31}$ hybrid interpenetrating networks, ${ }^{32}$ slip-link networks ${ }^{33}$ and homogeneous hydrogels ${ }^{34}$ have been introduced to toughen PAA hydrogels, resulting in improved mechanical properties. To the best of our knowledge, toughening hydrogels using chemical methods may limit their biomedical application as unreacted components adversely affect the biocompatibility. ${ }^{35,36}$ On the other hand although other methods were reported to enhance the mechanical properties of the hydrogels, some inherent disadvantages such as weak-self 
recovery and poor fatigue resistance still existed. ${ }^{29}$ Contribution of hyperbranched polymers to hydrogels' toughening performance physically leads to unconventional assemblies and complex structures with responsive properties. ${ }^{37,38}$

Recently, among the most extensively used nanoparticles, hyperbranched (HB) polymers, with a structure of highly branched tree-like dendritic material containing numerous - $\mathrm{OH}$ end groups, have been used in hydrogels. ${ }^{39-41}$ In a hydrogel crosslinked with HB polymers, polymeric chains have been held together by directionally discrete supramolecular binding sites to enhance the mechanical properties of the resultant composite. ${ }^{42,43}$ Evidence has suggested the potential for using HB polymers as a reinforcement agent in PAA based hydrogels. ${ }^{44,45}$

The aim of this study was to toughen PAA hydrogels with a combination of covalent and physical crosslinking by introducing $\mathrm{HB}$ polymers, without sacrificing the swelling ratio of the final product. This was achieved through in situ polymerisation, which is an easy process, and a cost-effective and environmentally friendly method. The structure-property relationship of the hydrogels was evaluated and a possible mechanism was also proposed.

\section{Material preparation and characterisation}

\section{Material preparation}

Acrylic acid monomer (AA), hyperbranched bis-MPA polyester64-hydroxyl (HB), ammonium persulfate (APS),the initiator, and $N, N^{\prime}$-methylenebis (acrylamide) (MBA), the crosslinking agent, were purchased of an analytical grade from sigma Aldrich (Australia) and used without any further purification. Sodium hydroxide ( $\mathrm{NaOH}$ ) was supplied by Chem-Supply (Australia). All aqueous solutions were prepared using Milli-Q water at room temperature.

Synthesis of the PAA-HB solution was done by following the method used in previous work. ${ }^{31}$ Briefly, $3.7 \mathrm{~g}$ of acrylic acid was dispersed in $3.5 \mathrm{~cm}^{3}$ of distilled water at $5{ }^{\circ} \mathrm{C}$. A $0.45 \mathrm{~g} \mathrm{~cm}^{-3}$ $\mathrm{NaOH}$ solution was prepared by dissolving $1.63 \mathrm{~g}$ of sodium hydroxide powder in $3.62 \mathrm{~g}$ of distilled water followed by cooling in an ice-water bath for $10 \mathrm{~min}$. The $\mathrm{NaOH}$ solution was then added to the acrylic acid solution dropwise with magnetic stirring at $5{ }^{\circ} \mathrm{C}$ for $20 \mathrm{~min}$. Finally, $0.025 \mathrm{~g}$ of MBA and $0.08 \mathrm{~g}$ of APS powder were added to the mixture, followed by vigorous shaking using an IKA Vortex 3 shaker to ensure homogeneity. The mixture was poured into a glass mould and placed in an oven (preset at $60^{\circ} \mathrm{C}$ ) for $30 \mathrm{~min}$ with the temperature being increased from 60 to $80{ }^{\circ} \mathrm{C}$ at a rate of $1{ }^{\circ} \mathrm{C} \mathrm{min}^{-1}$. After the overall heating duration of $30 \mathrm{~min}$, the prepared hydrogels were cooled to room temperature for $2 \mathrm{~h}$, and kept in the oven. The synthesized PAA samples were peeled off from the mould and stored in sealed bags at $4{ }^{\circ} \mathrm{C}$ for testing. For the PAA-HB sample, $3.5 \mathrm{~cm}^{3}$ of distilled water was first mixed with $0.6 \mathrm{~g}$ of HB powder and $3.7 \mathrm{~g}$ of acrylic acid was added to the suspension, which was then shaken vigorously using an IKA Vortex 3 shaker. The rest of the procedure was the same as above.

\section{Material characterisation}

Spectroscopic analysis. To investigate the possible interaction between the PAA and HB in the hybrid samples in terms of their functional groups, Fourier transform infrared (FTIR) spectroscopy (PerkinElmer Spectrum 400 spectrometer equipped with an attenuated total reflectance (ATR, top-plate type)) was performed. Each sample was mounted in turn onto the central crystal of the ATR plate, and air was used as a background reference before each scan. FTIR spectra were then recorded using 64 scans from 4000 to $550 \mathrm{~cm}^{-1}$ with a resolution of $4 \mathrm{~cm}^{-1}$ in absorbance mode with background subtraction.

Thermal analysis. The thermal behaviour of the hydrogels was studied using a differential scanning calorimeter (DSC) (TA instruments, USA). Samples were heated in a sealed aluminium crucible with another empty aluminium crucible as a reference, over the temperature range of $-30-300{ }^{\circ} \mathrm{C}$ at a rate of $10{ }^{\circ} \mathrm{C} \mathrm{min}^{-1}$ in a $\mathrm{N}_{2}$ atmosphere.

Morphology characterization. The surface morphology of the PAA and PAA-HB hydrogels was characterized using scanning electron microscopy (SEM) (CamScan MX2500). Dried specimens were mounted on aluminium stubs with double adhesive tape, then sputter coated with platinum at $2 \mathrm{~nm}$ thickness. The high voltage was set at $10 \mathrm{kV}$ and the distance from the sample to the beam source was kept constant for all the tests performed. To investigate the distribution of HB particles in the PAA hydrogel, a transmission electron microscope (TEM) (Philips CM200, the Netherlands) was employed. For preparation of the TEM specimens, all the hydrogel samples were completely dried in a vacuum oven overnight at $50{ }^{\circ} \mathrm{C}$. Then, the samples were ultramicrotomed using glass knives on an ultra-cut microtome (Leica Ultra cut-R ultramicrotome, Germany) to produce thin sections with a nominal thickness of $100 \mathrm{~nm}$.

Swelling behaviour. The swelling behaviour of the PAA and PAA-HB samples was tested by measuring the initial weight of the dry samples and subsequently the weight after they had been immersed in distilled water until equilibrium. Each test was repeated three times. Before measurement of the weight of the swollen samples, excess surface water was removed using filter paper. The swelling ratio was calculated using the equation:

$$
\mathrm{SR}=\frac{m_{t}-m_{0}}{m_{0}} \times 100
$$

where $m_{0}$ and $m_{t}$ are the weight of the sample in the initial dry and the swollen state, respectively.

Also, swelling kinetics of PAA and PAA-HB hydrogels for $\mathrm{pH}$ sensor applications were conducted in solutions at different $\mathrm{pH}$ values, i.e. 2, 7 and 10, attuned by sodium hydroxide and hydrochloric acid in deionized water. The dynamic swelling kinetics of both samples were explored by soaking and alternating them between solutions of $\mathrm{pH} 10$ and 2 every two hours, 3 times.

Mechanical properties. The mechanical properties of PAA and PAA-HB were measured for intact and notch-induced samples, using an Instron universal testing machine. All the samples were cut into a square shape with the dimensions $3.0 \mathrm{~mm} \times 3.0 \mathrm{~mm}$. Each sample's thickness was measured as 
$2.00 \pm 0.01 \mathrm{~mm}$ at five different points using a digital calliper. Notches with the depths of 3.0, 5.0, 8.0, 11.0 and $14.0 \mathrm{~mm}$ were introduced to the middle of the samples, using a laser cut machine. All samples (intact and notch-induced) were secured by sandpaper and superglue at both ends and the initial length of the samples was set at $5 \mathrm{~mm}$. The tests were conducted at room temperature with a strain rate of $50 \mathrm{~mm} \mathrm{~min}^{-1}$. All mechanical test results were the mean of at least five measurements. For dynamic testing, a PAA-HB sample was loaded to a certain stretch of $\lambda=4$ and unloaded to zero (first loading), after which the test was repeated at subsequent time intervals of $10 \mathrm{~s}, 10 \mathrm{~min}, 1 \mathrm{~h}$ and $24 \mathrm{~h}$ (second loading). Between the first and second loadings, all samples were wrapped in a polyethylene bag and stored at $4{ }^{\circ} \mathrm{C}$.

Statistical analysis. A univariate ANOVA with Bonferroni post-hoc was conducted (IBM SPSS Statistics for Windows, Version 22.0. Armonk, NY: IBM Corp.), having dependent variables of ultimate strength, elongation at break and toughness, and fixed factor of composition (PAA and $\mathrm{PAA} / \mathrm{HB}$ ) using an alpha of 0.05 .

\section{Results and discussion}

\section{Molecular interactions within hydrogels}

FTIR spectra of HB powder and PAA and PAA-HB hydrogels are shown in Fig. 1. The peaks observed at $1728 \mathrm{~cm}^{-1}$ and $1440 \mathrm{~cm}^{-1}$ are ascribed to $\mathrm{H}$-bonded ester $\mathrm{C}=\mathrm{O}$ groups and COO-stretching of the HB polymer, as shown in Fig. 1(a). ${ }^{31}$ The characteristic absorption peaks of $\mathrm{C}-\mathrm{O}(-\mathrm{OH})$ stretching hydroxyl and $\mathrm{O}-\mathrm{C}$ stretching ester groups occur at $1041 \mathrm{~cm}^{-1}$ and $1121 \mathrm{~cm}^{-1}$, respectively. For the PAA hydrogel, peaks at 1680, 1551 and $1403 \mathrm{~cm}^{-1}$ are related to the $-\mathrm{C}=\mathrm{O}$ asymmetric stretching of $-\mathrm{COOH}$ and $\mathrm{COO}^{-}$groups, and $\mathrm{COO}$ symmetric stretching of $-\mathrm{COO}^{-}$groups, respectively. ${ }^{46,47}$ Stretching of $-\mathrm{CH}_{2}-$ (asymmetric and symmetric) and bending of $\mathrm{C}-\mathrm{H}$ were observed at 2939, 2855, and $1450 \mathrm{~cm}^{-1}$, indicating the existence of PAA main chains. ${ }^{48}$ During the neutralization process of PAA by $\mathrm{NaOH},-\mathrm{COOH}$ groups of AA were partially converted into - COONa, as shown in Fig. 1(b). For the PAA-HB, shown in Fig. 1(c), with the addition of HB into the PAA structure, compared to the PAA curves, new absorption peaks due to the $\mathrm{C}-\mathrm{O}(-\mathrm{OH})$ hydroxyl and $\mathrm{O}-\mathrm{C}$ ester groups of $\mathrm{HB}$ occurred at 1041 and $1121 \mathrm{~cm}^{-1}$, attributed to the polymer binder. ${ }^{49}$ These characteristic absorption peaks provide evidence for the successful preparation of the PAA-HB hydrogel.

The molecular interaction of PAA and PAA-HB hydrogels was also investigated by DSC measurements from -50 to $75{ }^{\circ} \mathrm{C}$, as shown in Fig. 2. From the DSC curve, it can be seen that the addition of the $\mathrm{HB}$ polymer into the PAA results in a reduction in the glass transition temperature $\left(T_{\mathrm{g}}\right)$ from -0.63 to $-7.43{ }^{\circ} \mathrm{C}$. This phenomenon is attributed to the supramolecular structure of HB, which can cause physical entanglement between PAA network structures and increase the crystallinity of the final hydrogel as compared to those features in the PAA hydrogel. This result confirmed that the contribution of $\mathrm{HB}$ can cause the formation of PAA-based hybrid materials, in which mechanical and other properties could also be changed. ${ }^{4}$

\section{Morphological understanding}

To confirm the interactions between the HB polymer and the PAA, the morphologies of the hydrogels were observed by SEM at different scales. The $\mathrm{HB}$ polymer binders were evenly

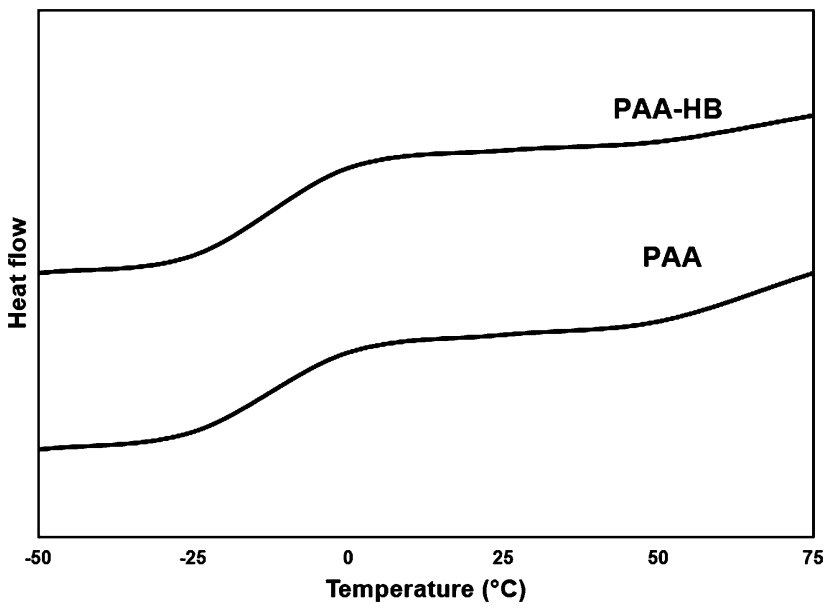

Fig. 2 DSC curves of PAA and PAA hybrid samples.

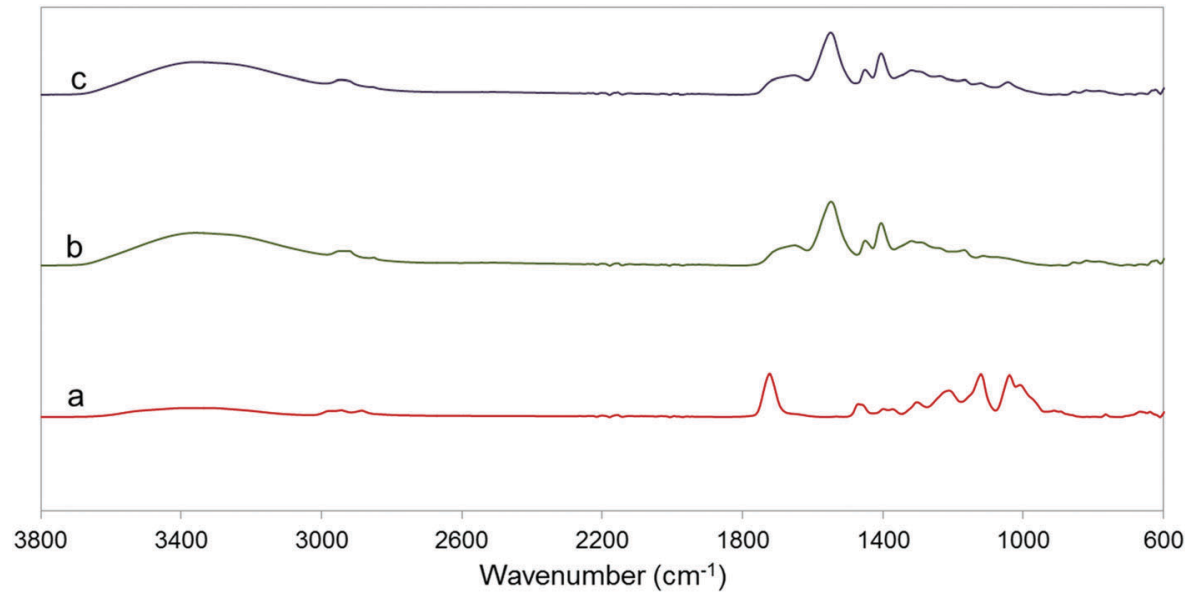

Fig. 1 FTIR spectra of (a) HB powder, (b) PAA and (c) PAA-HB hydrogels. 


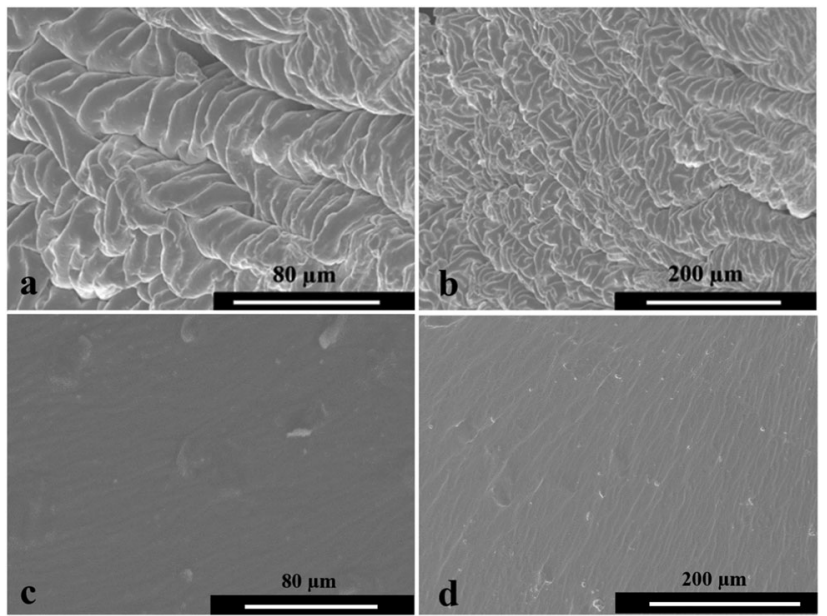

Fig. 3 SEM images of (a) and (b) PAA-HB surfaces and (c) and (d) PAA surfaces.

distributed into the PAA structure, as shown in Fig. 3. The rough surface of the PAA-HB also suggested good dispersion of the $\mathrm{HB}$ within the PAA. A change in surface morphology of the PAA-HB samples, as compared to the smooth surface of a PAA sample, is consistent with FTIR results that suggest the possibility of hyperbranched polymer contribution to the structural organization of PAA molecules. Also TEM images suggest good dispersion of HB inside the PAA, as shown below.

TEM images of PAA-HB samples are shown in Fig. 4. The aggregated nanospheres in the range of 10-200 $\mathrm{nm}$ in diameter are attributed to the HB polymer binders which are dispersed well in the PAA matrix. Due to the numerous hydroxyl groups at the ends of the $\mathrm{HB}$, the particles tend to aggregate. Meanwhile, the hydrophilic character of these hydroxyl groups could help to enhance the compatibility of $\mathrm{HB}$ with PAA during in situ polymerisation. $^{31}$

\section{Water swelling behaviours}

The swelling behaviours of PAA and PAA-HB hydrogels immersed in water at $25{ }^{\circ} \mathrm{C}$ are shown in Fig. 5. The addition of $\mathrm{HB}$ into the PAA solution caused increased water uptake by the hydrogel. In the first 15 minutes, the water uptake of both hydrogels was extremely fast, followed by constant increments, finally reaching equilibrium within $150 \mathrm{~min}$. In the PAA sample, the swelling ratio (SR) at the equilibrium stage was about $86 \mathrm{k} \%$ of its initial weight. With the introduction of $\mathrm{HB}$, however, this amount increased to a higher value of $9.6 \mathrm{k} \%$, which was about 1.12 times higher than that of the PAA. This increase was attributed to the availability of a large

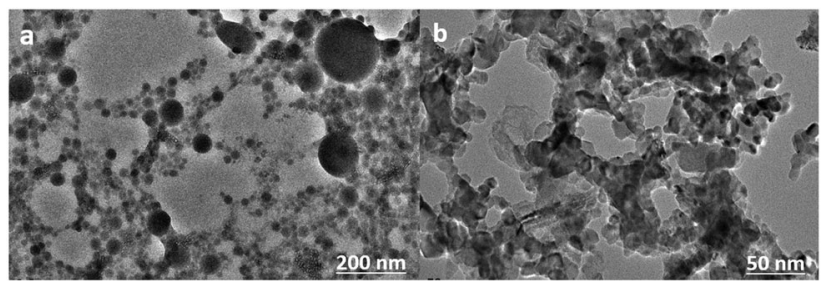

Fig. 4 TEM images of the PAA-HB hydrogel.

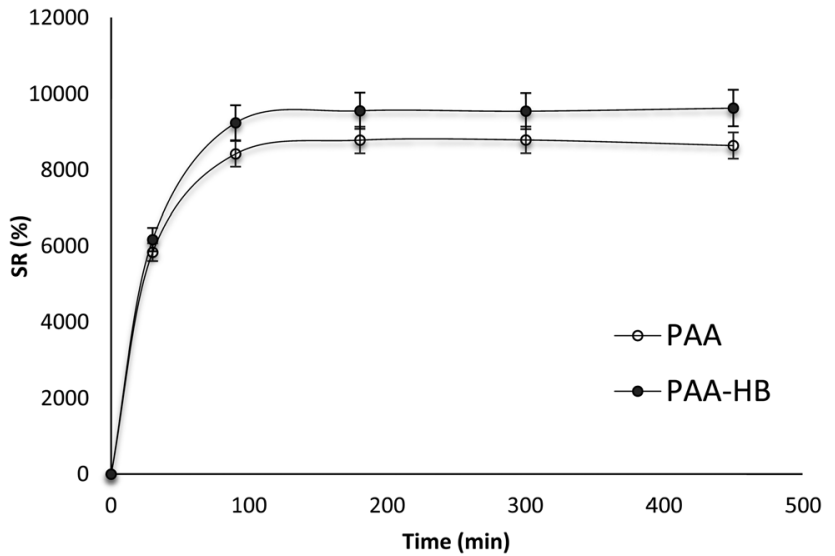

Fig. 5 Swelling ratios of PAA and PAA-HB hydrogels immersed in water.

number of hydrophilic hydroxyl groups in the HB particles, which could bind to water and increase the absorption capacity of the hydrogel. The slopes of SR versus immersion duration of both PAA and PAA-HB samples had similar trends. Once the time exceeded $150 \mathrm{~min}$, the SR of samples reached a plateau, indicating excellent water retention by the PAA and PAA-HB hydrogels.

\section{Mechanical behaviours of hydrogels}

The effects of adding HB to the PAA structure on the mechanical properties of the PAA-HB hydrogel are shown in Fig. 6. As shown, the ultimate tensile strength of the PAA-HB hydrogel improved by $130 \%(1.15 \pm 0.10 \mathrm{MPa})$ compared to the PAA hydrogel with an ultimate strength of approximately $0.50 \pm 0.04$ $\mathrm{MPa}$. Also, elongation at break is doubled, from $340 \pm 12 \%$ for PAA to $650 \pm 35 \%$ for PAA-HB. Thus, the fracture energy of the PAA-HB hydrogel, measured as $4483 \pm 39 \mathrm{~kJ} \mathrm{~m}^{-3}$, is approximately four times greater than that of the PAA hydrogel, which was $1020 \pm 29 \mathrm{~kJ} \mathrm{~m}^{-3}$.

Stress-strain curves for the PAA-HB samples at different stretch ratios are presented in Fig. 7. The stretch ratio $(\lambda)$ was defined as the ratio of final to initial length. As is evident, the stress-strain relationship for all stretch ratios is linear for strain less than $50 \%$. As $\lambda$ increases, the ultimate tensile stress and the corresponding strain both increase. Table 1 shows the change in ultimate stress, the Young's modulus and toughness at different stretch ratios.

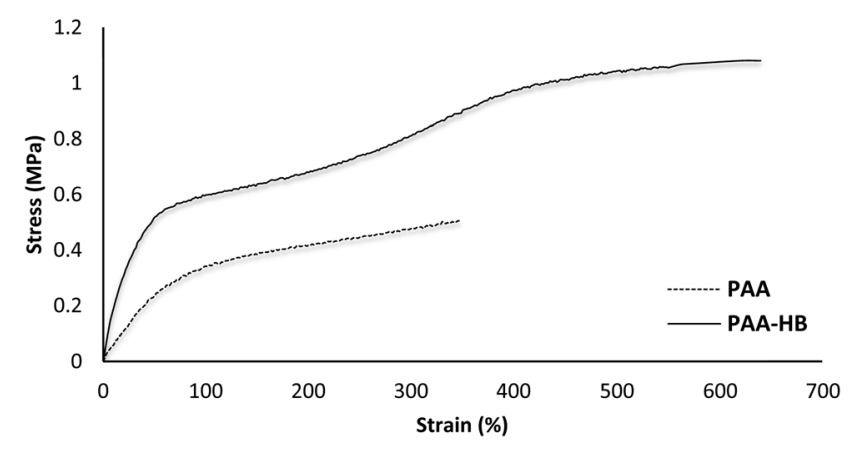

Fig. 6 Mechanical properties of PAA and PAA-HB hydrogels. 


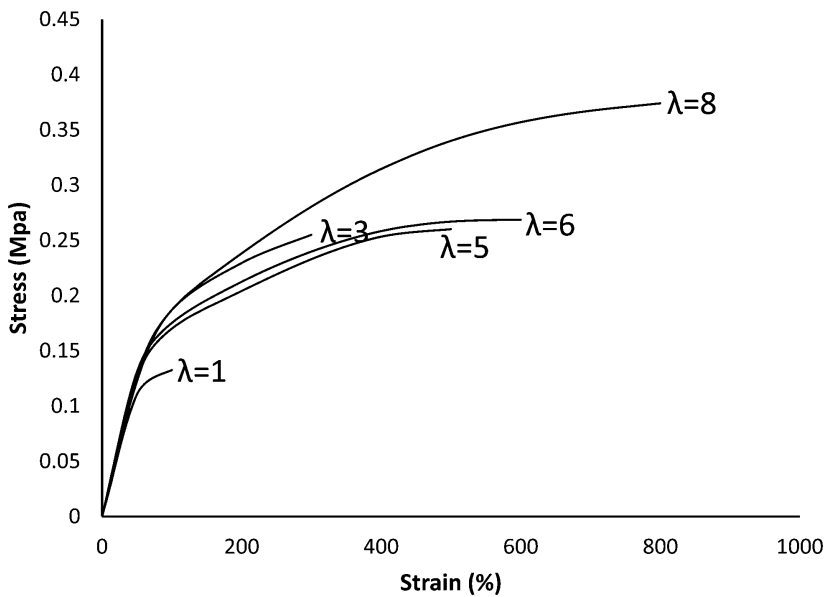

Fig. 7 Stress-strain relationship for PAA-HB hydrogels at different stretch ratios.

A comparison of the mechanical properties of PAA and PAA-HB at different notch depths is presented in Fig. 8. The stretchability of PAA-HB samples with selected notches of 0 and $14 \mathrm{~mm}$ is also shown in Fig. 8.

As shown, the unnotched samples display greater toughness, elongation at break and ultimate strength than the notched samples. Also the PAA-HB samples have significantly enhanced mechanical properties compared to the PAA samples. Surprisingly, the ultimate tensile strength for the PAA-HB samples with notch depths of 3, 5 and $8 \mathrm{~mm}$ remains constant at approximately $0.10 \pm$ $0.02 \mathrm{MPa}$. A similar trend is found for the samples with 11 and $14 \mathrm{~mm}$ notches, as shown in Fig. 8(a). Generally, the elongation of the unnotched PAA-HB samples is about twice that of the intact PAA samples. The stretchability of the PAA-HB and PAA samples for different notch depths is about $20 \%$ and $50 \%$ lower than those of the corresponding intact samples, respectively, and remains constant for all notch depths, as shown in Fig. 8(b). Fig. 8(c) shows that both intact and notched PAA-HB samples with different depths have significant improvement in fracture toughness compared with the corresponding PAA samples. The PAA samples with notch depths greater than $5 \mathrm{~mm}$ have negligible toughness.

Statistical analysis revealed that the overall effect of introducing different notches on the selected mechanical properties of stiffness, elongation at break and ultimate strength of both PAA and PAA-HB was significant $(P<0.01)$. Due to the marked decrease in these mechanical properties of PAA after notch insertion, changes in notch depth showed no significant effect on the ultimate strength $(P=0.99)$, whereas in the PAA-HB samples, introducing a notch with depth greater than $11 \mathrm{~mm}$ resulted in a significant change in ultimate strength $(p=0.004)$.
Meanwhile, it was found that a change in notch depth from 1 to $5 \mathrm{~mm}$ had a significant effect on the PAA samples' elongation at break $(P=0.003)$, but not when the notch was greater than $5 \mathrm{~mm}$ $(P=1.00)$. In contrast, the addition of HB into the PAA structure improved the elongation at break property. It was shown that the change was negligible for samples with notch depth difference less than $6 \mathrm{~mm}(P=0.593)$. For PAA samples with notch depth greater than $2 \mathrm{~mm}$, the change in fracture toughness was significant $(P=0.021)$. However, notches of at least $6 \mathrm{~mm}$ or greater needed to be introduced to the PAA-HB samples to significantly affect toughness $(P=0.004)$.

Fig. 9 shows the results of dynamic tests that were performed on intact PAA-HB samples. As shown, the hysteresis effect for the first loading to a stretch of $\lambda=4$ and the subsequent unloading to zero is huge in comparison to that of samples with a second loading. The PAA-HB hydrogels dissipated energy effectively and their hysteresis loop was reduced for the second loading at different time intervals after the first loading. It seems that the change in maximum stress at a stretch ratio of 4 was negligible for all second tests for a given sample, indicating the effect of hyperbranching on the mechanical properties of the PAA due to increasing physical interaction or internal friction.

\section{Proposed mechanism}

Hydrogels of PAA and PAA-HB were successfully synthesized through a one-step, cost-effective and environmentally friendly method. From the above investigations, the mechanism for the PAA-HB network structure can be proposed. Fig. 10 illustrates the structure of the PAA hydrogel with HB particles. Numerous $-\mathrm{COOH}$ groups of PAA result in the formation of hydrogen bonding. These hydrogen bond formations are increased in the presence of water. On the other hand, HB experiences some physical entanglement in PAA networks due to its high molecular weight and star shape. Meanwhile, the $-\mathrm{OH}$ groups of $\mathrm{HB}$ can effectively connect with the $-\mathrm{COOH}$ groups of PAA, resulting in the formation of hydrogen bonding. After MBA and APS were added during in situ polymerisation, PAA networks were constructed tightly around the HB molecules. Therefore, PAA-HB hydrogels are expected to have higher mechanical properties compared to those of PAA hydrogels.

To compare our study with other research studies relevant information is presented in Table 2 , more details have to be noticed during the comparison as mechanical characterisation was performed under different conditions. As it can be seen from the table, different toughening agents/methods, i.e. graphene oxide, branched polymers, hyper-branched polymers, IPN, etc., may improve some mechanical properties while their negative effects on other properties, such as swelling ability need to be addressed.

Table 1 Change in the selected mechanical properties of PAA-HB at different stretch ratios

\begin{tabular}{|c|c|c|c|c|c|c|}
\hline \multirow[b]{2}{*}{ Mechanical properties } & \multirow[b]{2}{*}{ Unit } & \multicolumn{5}{|c|}{$\underline{\text { Stretch ratio }(\lambda)}$} \\
\hline & & 1 & 3 & 5 & 6 & 8 \\
\hline Ultimate stress & $\mathrm{MPa}$ & $0.13 \pm 0.21$ & $0.25 \pm 0.18$ & $0.26 \pm 0.27$ & $0.27 \pm 0.15$ & $0.37 \pm 0.14$ \\
\hline Young's modulus & $\mathrm{MPa}$ & $2.21 \pm 0.18$ & $2.55 \pm 0.12$ & $2.55 \pm 0.08$ & $2.55 \pm 0.16$ & $2.55 \pm 0.21$ \\
\hline Toughness & $\mathrm{kJ} \mathrm{m}^{-3}$ & $97 \pm 11$ & $459 \pm 32$ & $835 \pm 49$ & $1094 \pm 69$ & $2124 \pm 51$ \\
\hline
\end{tabular}



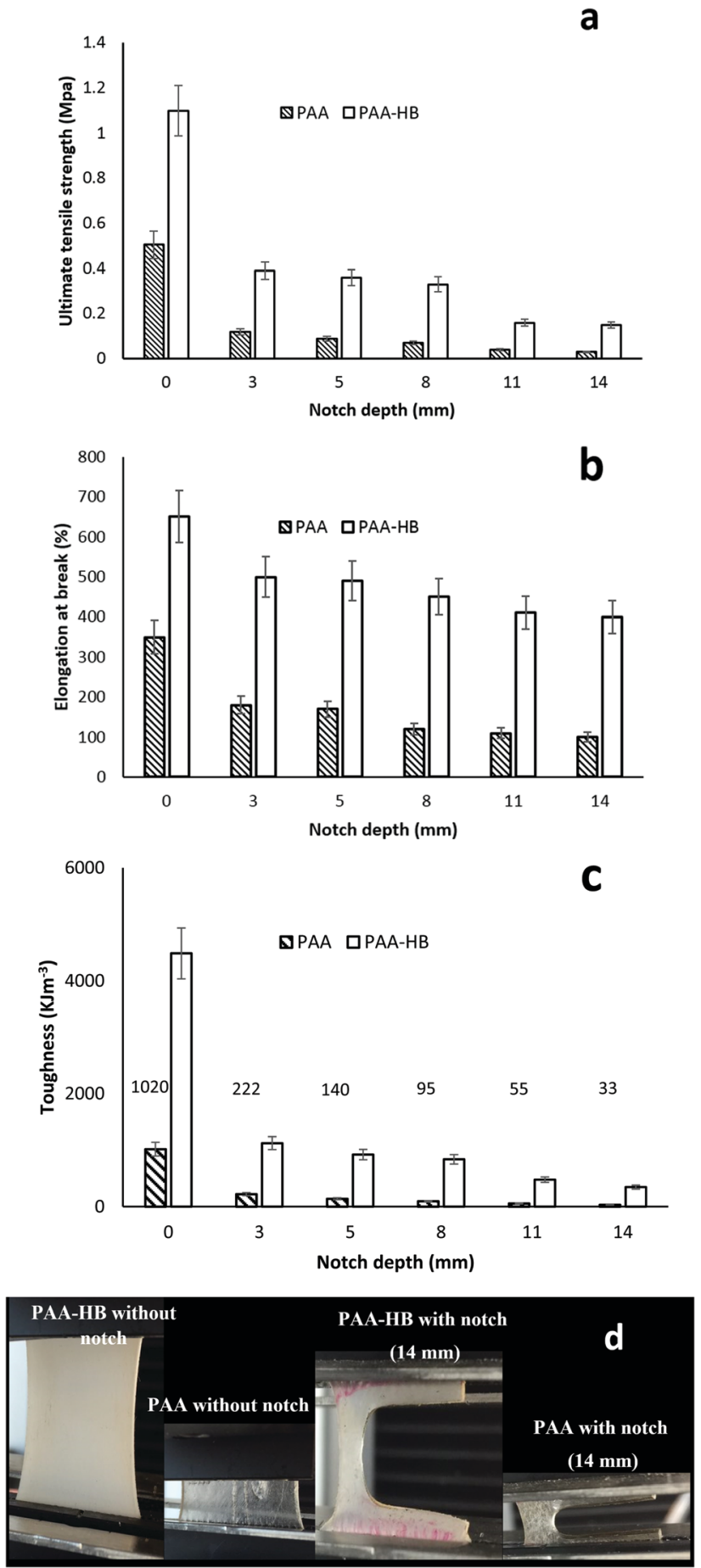

Fig. 8 Comparison of the mechanical properties of PAA with PAA-HB hydrogel for different notch depths. (a) Ultimate tensile strength, (b) elongation at break and (c) toughness. Figure (d) shows the stretchability of the intact sample (un-notched) versus the notched sample $(14 \mathrm{~mm})$ for both PAA and PAA-HB hydrogels.

\section{Sensor application}

To investigate the possibility of utilizing the toughened PAA-HB hydrogel as a pH-sensitive sensor, its enviro-responsive and dynamic swelling properties were investigated here. Measuring the swelling ratio as a function of time in solutions at different

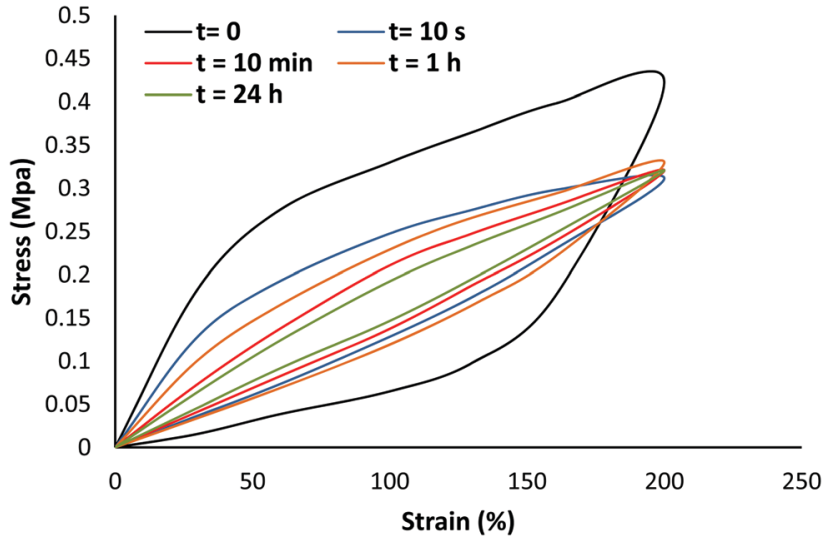

Fig. 9 Hysteresis effect of loading to a certain stretch $(\lambda=4)$ and unloading to zero for first and second loadings with different time intervals. All dynamic tests were performed at $50 \mathrm{~mm} \mathrm{~min}^{-1}$ strain rate.

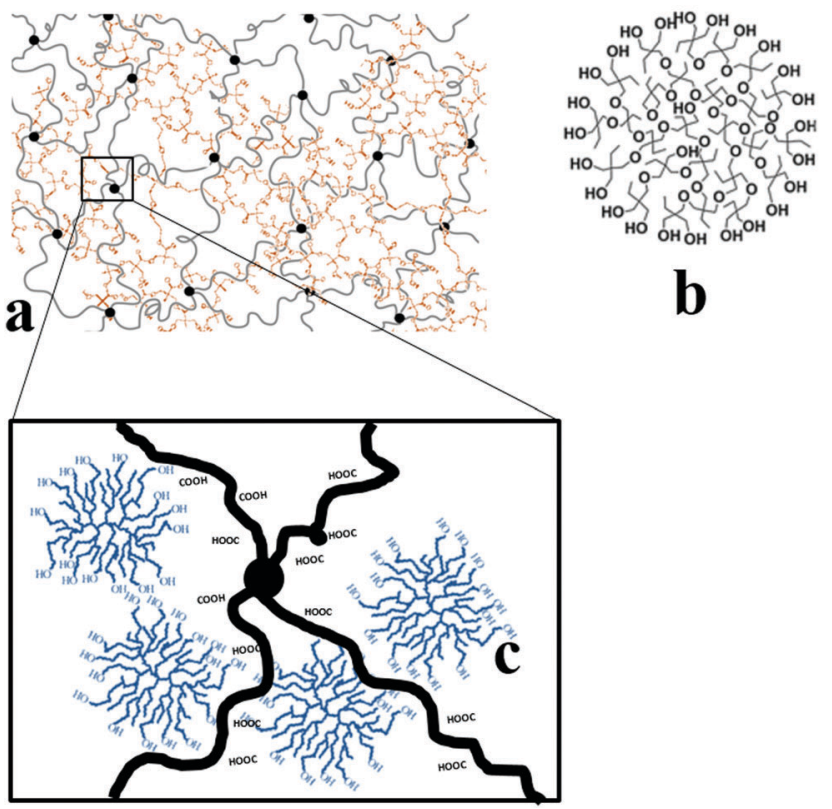

Fig. 10 (a) Interpenetrating networks of PAA-HB; (b) schematic structure of a HB molecule; and (c) schematic illustration of the possible physical interaction and hydrogen bond formation mechanism between PAA chains and $\mathrm{HB}$ molecules.

$\mathrm{pH}$ values of 2,7 and 10 revealed that the overall trend of swelling kinetic curves for PAA-HB samples are consistent with those without HB. Below the $\mathrm{p} K_{\mathrm{a}}$, both PAA and PAA-HB samples swelled significantly less than those above the $\mathrm{p} K_{\mathrm{a}}$ $(P=0.041)$. However, the increase of the $\mathrm{pH}$ value resulted in a higher swelling ratio for PAA-HB samples compared to that of PAA samples (Fig. 11a). As it is shown in Fig. 11b, despite a negligible change in the equilibrium swelling ratio (ESR) for PAA and PAA-HB samples at $\mathrm{pH}=3$, increasing the $\mathrm{pH}$ value from 7 to 10 resulted in $10 \%$ and $23 \%$ ESR increases for PAA-HB compared to those of PAA, respectively.

Dynamic swelling behavior, with the $\mathrm{pH}$ value alternating between 2 and 10, was examined to confirm the reversibility of 
Table 2 Different PAA hydrogel toughening methods with selected mechanical and swelling properties

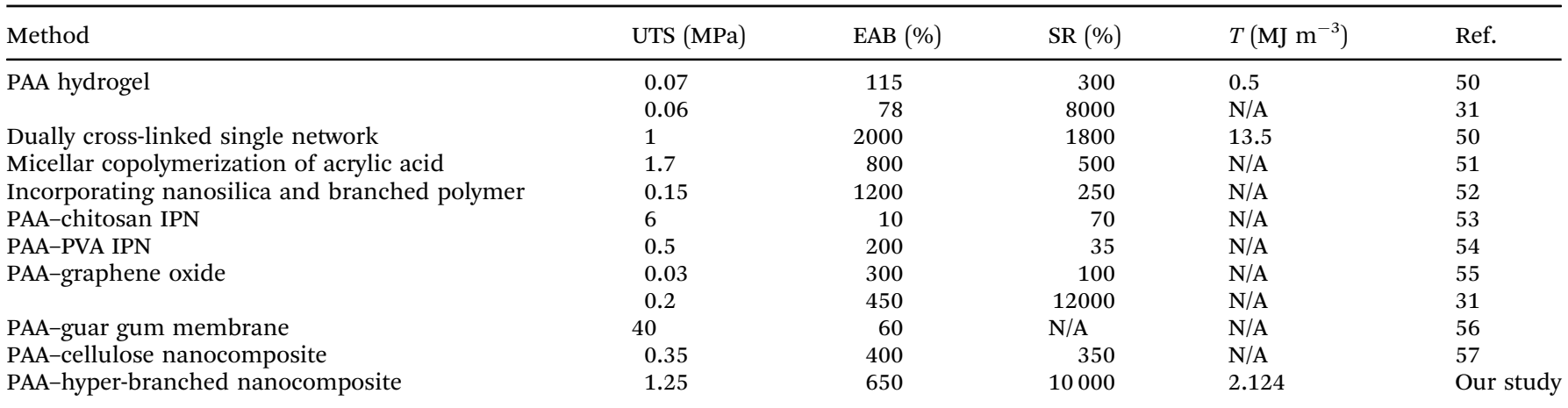

the swelling process and the ability of the samples to maintain their swelling capacity during cycling deformation. All cycles were performed in two-hour time intervals as swelling kinetics showed that samples approximately approached their equilibrium state after two hours. The ratio of samples' swelling ratio at pH 10 and 2 (mentioned as SR10/SR2 and called the reversibility factor (RF)) was calculated and compared for three cycles. As shown in Fig. 12a, comparing the 2nd and 3rd cycles to the 1st cycle, changes in RF for PAA-HB samples were significantly lower than those of PAA samples $(P=0.046)$. It means that differences between the swelling ratio at $\mathrm{pH} 10$ and 2 were less for PAA-HB samples as compared to those of PAA samples.
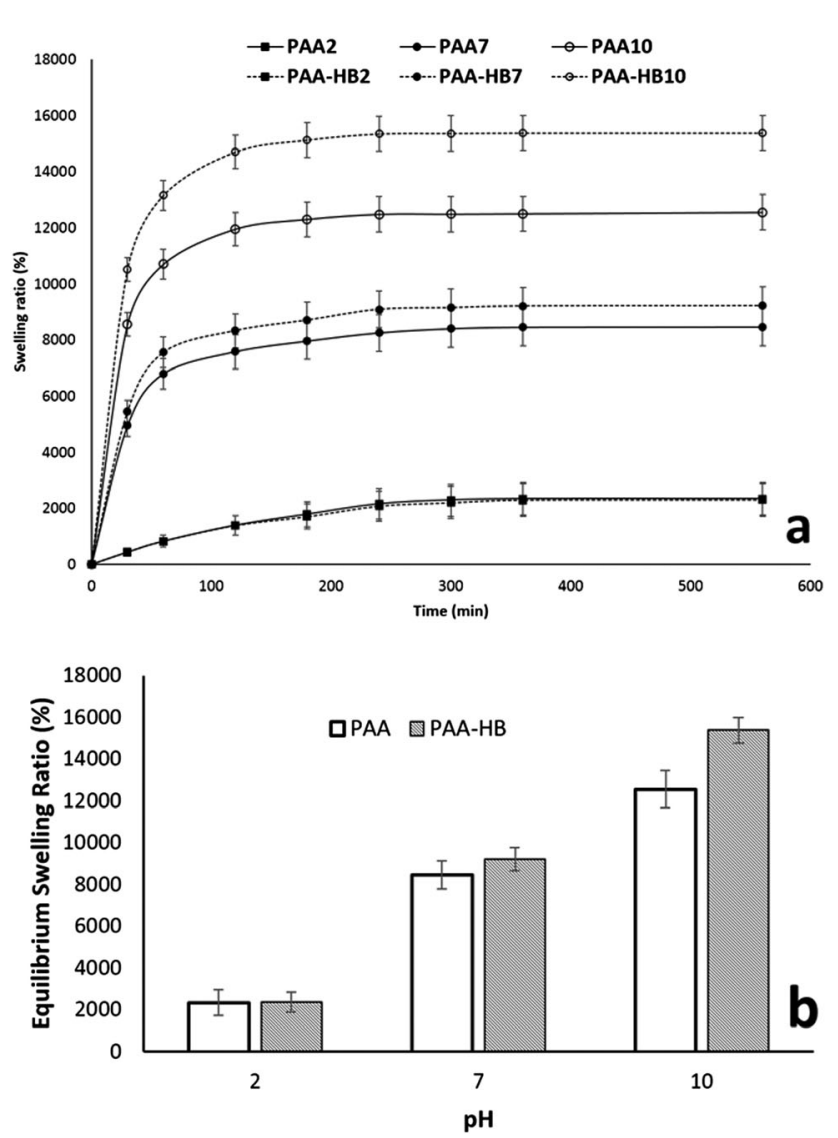

Fig. 11 Effect of $\mathrm{pH}$ values on the (a) swelling kinetics and (b) equilibrium swelling ratio (ESR) of PAA and PAA-HB samples.
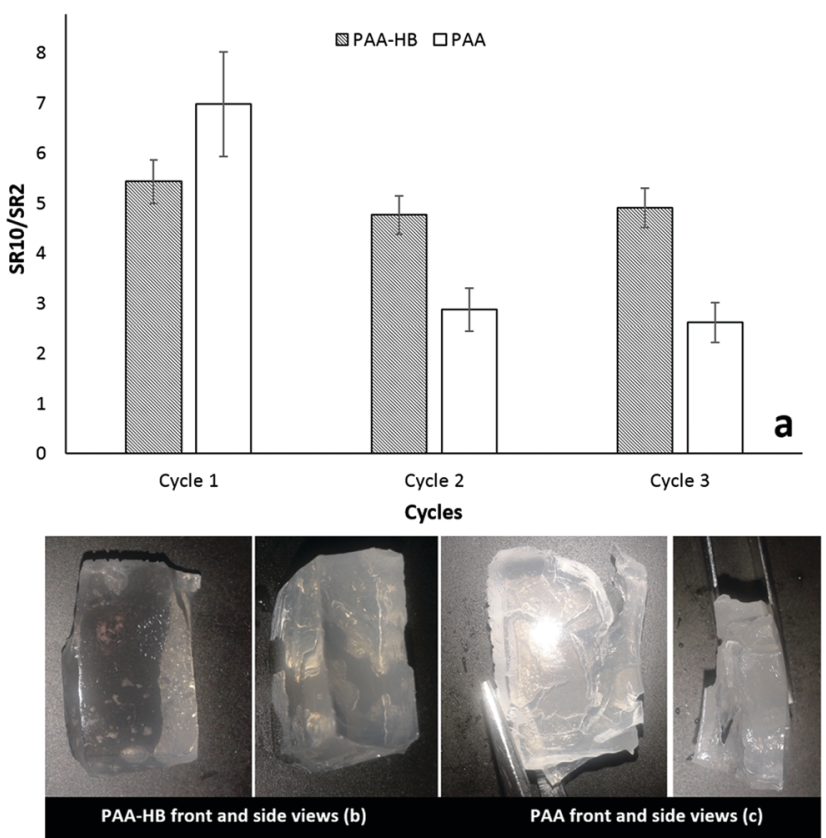

Fig. 12 (a) Change in the SR10/SR2 ratio during three cycles of dynamic swelling revealed higher $\mathrm{pH}$ sensitivity of PAA-HB hydrogels compared to that of PAA samples. After 3 cycles, the better dimensional stability of (b) PAA-HB samples compared to that of (c) PAA samples was in general agreement with the enhanced mechanical properties from $\mathrm{HB}$.

Meanwhile, after performing 3 cycles of dynamic swelling, PAA-HB samples were more structurally stable than PAA hydrogels. Deep cracks and dimensional instability were observed in PAA samples after the alternative swelling/de-swelling process. HB addition provides improved mechanical properties to the hydrogel, enhances the $\mathrm{pH}$ sensitivity of PAA hydrogels and improves the swelling properties with a positive impact on dimensional stability.

\section{Conclusions}

In this study, by introducing a HB polymer into PAA using in situ polymerisation, a hydrogel network was formed with the mechanical entanglement of $\mathrm{HB}$ into the PAA structure and a possible hydrogen bond between the active group of $\mathrm{HB}$ and $-\mathrm{COOH}$ groups of PAA. TEM results showed that spherical HB 
particles were well dispersed in the PAA matrix, indicating good compatibility of PAA with HB. Significant improvements in the mechanical properties were achieved when the capability of water uptake was increased by approximately $20 \%$. With the introduction of notches in the PAA and PAA-HB samples, the effects on selected mechanical properties were significant; but notches of at least $6 \mathrm{~mm}$ or greater had to be introduced to significantly affect the toughness of the PAA-HB samples. It is worth mentioning the possibility of their application in sensors, where the simultaneous enviro-responsive and mechanical properties of hydrogels are important.

\section{Acknowledgements}

Y. Tang is grateful for the support of the Australian Research Council (ARC) with a Discovery Early Career Research Award (DECRA) Grant (DE120102784) for the research work.

\section{References}

1 L. Brannon-Peppas and N. A. Peppas, Equilibrium swelling behavior of pH-sensitive hydrogels, Chem. Eng. Sci., 1991, 46(3), 715-722.

2 J. Esmaiel, T. Javad and S. S. Alireza, Swelling characteristics of acrylic acid polyelectrolyte hydrogel in a dc electric field, Smart Mater. Struct., 2007, 16(5), 1614.

3 Z. Lin, D. Mei, M. Chen, Y. Wang, X. Chen, Z. Wang, B. He, H. Zhang, X. Wang, W. Dai, Y. Yin and Q. Zhang, A comparative study of thermo-sensitive hydrogels with waterinsoluble paclitaxel in molecule, nanocrystal and microcrystal dispersions, Nanoscale, 2015, 7(36), 14838-14847.

4 R. Peng, Y. Yu, S. Chen, Y. Yang and Y. Tang, Conductive nanocomposite hydrogels with self-healing property, $R S C A d v$., 2014, 4(66), 35149-35155.

5 L. Han, X. Lu, M. Wang, D. Gan, W. Deng, K. Wang, L. Fang, K. Liu, C. W. Chan, Y. Tang, L. Wen and H. Yuan, A musselinspired conductive, self-adhesive, and self-healable tough hydrogel as cell stimulators and implantable bioelectronics, Small, 2017, 13(2), DOI: 10.1002/smll.201601916.

6 I. Hamed, F. Özogul and J. M. Regenstein, Industrial applications of crustacean by-products (chitin, chitosan, and chitooligosaccharides): A review, Trends Food Sci. Technol., 2016, 48, 40-50.

7 N. Eslahi, M. Abdorahim and A. A. Simchi, Smart polymeric hydrogels for cartilage tissue engineering: A review on the chemistry and biological functions, Biomacromolecules, 2016, 17(11), 3441-3463.

8 J. Wang, J. A. Kaplan, Y. L. Colson and M. W. Grinstaff, Mechanoresponsive materials for drug delivery: Harnessing forces for controlled release, Adv. Drug Delivery Rev., 2016, 108, 68-82.

9 R. Singhal and K. Gupta, A review: Tailor-made hydrogel structures (classifications and synthesis parameters), Polym.-Plast. Technol. Eng., 2016, 55(1), 54-70.

10 S. L. Lim, W. N. H. Tang, C. W. Ooi, E. S. Chan and B. T. Tey, Rapid swelling and deswelling of semi-interpenetrating network poly (acrylic acid)/poly (aspartic acid) hydrogels prepared by freezing polymerization, J. Appl. Polym. Sci., 2016, 133(24), DOI: 10.1002/app43515.

11 M. Zhong, Y. Liu, X. Liu, F. Shi, L. Zhang, M. Zhu and X. Xie, Dually cross-linked single network poly (acrylic acid) hydrogels with superior mechanical properties and water absorbency, Soft Matter, 2016, 12, 5420-5428.

12 A. Pal, A. Giri and A. Bandyopadhyay, Influence of hydrodynamic size and zeta potential of a novel polyelectrolyte poly (acrylic acid) grafted guar gum for adsorption of $\mathrm{Pb}$ (II) from acidic waste water, J. Environ. Chem. Eng., 2016, 4(2), 1731-1742.

13 S. Sharma, A. Dua and A. Malik, Superabsorbent polymer gels based on polyaspartic acid and polyacrylic acid, J. Mater. Sci. Eng., 2016, 5, 235.

14 Y. Chen, Y. Zhang, F. Wang, W. Meng, X. Yang, P. Li, J. Jiang, H. Tan and Y. Zheng, Preparation of porous carboxymethyl chitosan grafted poly (acrylic acid) superabsorbent by solvent precipitation and its application as a hemostatic wound dressing, Mater. Sci. Eng., C, 2016, 63, 18-29.

15 J. E. Elliott, M. Macdonald, J. Nie and C. N. Bowman, Structure and swelling of poly (acrylic acid) hydrogels: effect of $\mathrm{pH}$, ionic strength and dilution on the crosslinked polymer structure, Polymer, 2004, 45(5), 1503-1510.

16 N. A. Peppas and J. J. Sahlin, Hydrogels as mucoadhesive and bioadhesive materials: a review, Biomaterials, 1996, 17(16), 1553-1561.

17 B. Işılk, Swelling behavior and determination of diffusion characteristics of acrylamide-acrylic acid hydrogels, J. Appl. Polym. Sci., 2004, 91(2), 1289-1293.

18 M. J. Yin, M. Yao, S. Gao, A. Zhang, H. Tam and P. A. Wai, Rapid 3D patterning of poly (acrylic acid) ionic hydrogel for miniature pH sensors, Adv. Mater., 2016, 28(7), 1394-1399.

19 J. You, S. Xie, J. Cao, H. Ge, M. Xu, L. Zhang and J. Zhou, Quaternized chitosan/poly (acrylic acid) polyelectrolyte complex hydrogels with tough, self-recovery, and tunable mechanical properties, Macromolecules, 2016, 49(3), 1049-1059.

20 J. Li, Z. Su, X. Ma, H. Xu, Z. Shi, J. Yin and X. Jiang, In situ polymerization induced supramolecular hydrogels of chitosan and poly (acrylic acid-acrylamide) with high toughness, Mater. Chem. Front., 2017, 1, 310-318.

21 L. Yang and C. Zhang, Highly stretchable and self-healing hydrogels based on poly (acrylic acid) and functional POSS, Chin. J. Polym. Sci., 2016, 34(2), 185-194.

22 M. A. Haq, Y. Su and D. Wang, Mechanical properties of PNIPAM based hydrogels: A review, Mater. Sci. Eng., C, 2017, 70, 842-855.

23 G. He, W. Ke, X. Chen, Y. Kong, H. Zheng, Y. Yin and W. Cai, Preparation and properties of quaternary ammonium chitosan- $g$ poly(acrylic acid-co-acrylamide) superabsorbent hydrogels, React. Funct. Polym., 2017, 111, 14-21.

24 K. Haraguchi, Synthesis and properties of soft nanocomposite materials with novel organic/inorganic network structures, Polym. J., 2011, 43(3), 223-241.

25 K. S. Anseth, C. N. Bowman and L. Brannon-Peppas, Mechanical properties of hydrogels and their experimental determination, Biomaterials, 1996, 17(17), 1647-1657. 
26 D. Kim and K. Park, Swelling and mechanical properties of superporous hydrogels of poly(acrylamide-co-acrylic acid)/ polyethylenimine interpenetrating polymer networks, Polymer, 2004, 45(1), 189-196.

27 Q. Su, L. Duan, M. Zou, X. Chen and G. H. Gao, The tough allograft adhesive behavior between polyacrylamide and poly(acrylic acid) hydrophobic association hydrogels, Mater. Chem. Phys., 2017, 193, 57-62.

28 M. Zeng, Z. Feng, Y. Huang, J. Liu, J. Ren, Q. Xu and L. Fan, Chemical structure and remarkably enhanced mechanical properties of chitosan-graft-poly(acrylic acid)/polyacrylamide double-network hydrogels, Polym. Bull., 2017, 74(1), 55-74.

29 X. Liu, L. Duan and G. Gao, Rapidly self-recoverable and fatigue-resistant hydrogels toughened by chemical crosslinking and hydrophobic association, Eur. Polym. J., 2017, 89, 185-194.

30 P. Thoniyot, M. J. Tan, A. A. Karim, D. J. Young and X. J. Loh, Nanoparticle-hydrogel composites: Concept, design, and applications of these promising, multi-functional materials, Adv. Sci., 2015, 2(1-2), DOI: 10.1002/advs.201400010.

31 Y. Yu, L. C. X. De Andrade, L. Fang, J. Ma, W. Zhang and Y. Tang, Graphene oxide and hyperbranched polymertoughened hydrogels with improved absorption properties and durability, J. Mater. Sci., 2015, 50(9), 3457-3466.

32 S. Naficy, S. Kawakami, S. Sadegholvaad, M. Wakisaka and G. M. Spinks, Mechanical properties of interpenetrating polymer network hydrogels based on hybrid ionically and covalently crosslinked networks, J. Appl. Polym. Sci., 2013, 130(4), 2504-2513.

33 S. Naficy, H. R. Brown, J. M. Razal, G. M. Spinks and P. G. Whitten, Progress toward robust polymer hydrogels, Aust. J. Chem., 2011, 64(8), 1007-1025.

34 X. Cai, H. Tong, X. Shen, W. Chen, J. Yan and J. Hu, Preparation and characterization of homogeneous chitosanpolylactic acid/hydroxyapatite nanocomposite for bone tissue engineering and evaluation of its mechanical properties, Acta Biomater., 2009, 5(7), 2693-2703.

35 M. I. Baker, S. P. Walsh, Z. Schwartz and B. D. Boyan, A review of polyvinyl alcohol and its uses in cartilage and orthopedic applications, J. Biomed. Mater. Res., Part B, 2012, 100(5), 1451-1457.

36 H. Park and K. Park, Biocompatibility issues of implantable drug delivery systems, Pharm. Res., 1996, 13(12), 1770-1776.

37 E. Schwab and S. Mecking, Synthesis and properties of highly branched polycations with an aliphatic polyether scaffold, J. Polym. Sci., Part A: Polym. Chem., 2005, 43(19), 4609-4617.

38 J. Yuan, S. Soll, M. Drechsler, A. H. E. Muller and M. Antonietti, Self-assembly of poly(ionic liquid)s: Polymerization, mesostructure formation, and directional alignment in one step, J. Am. Chem. Soc., 2011, 133(44), 17556-17559.

39 A. Klymenko, T. Nicolai, L. Benyahia, C. Chassenieux, O. Colombani and E. Nicol, Multiresponsive hydrogels formed by interpenetrated self-assembled polymer networks, Macromolecules, 2014, 47(23), 8386-8393.

40 J. B. Lena, A. K. Goroncy, J. J. Thevarajah, A. R. Maniego, G. T. Russell, P. Castignolles and M. Gaborieau, Effect of transfer agent, temperature and initial monomer concentration on branching in poly(acrylic acid): A study by ${ }^{13} \mathrm{C}$ NMR spectroscopy and capillary electrophoresis, Polymer, 2017, 114, 209-220.

41 P. Castignolles, R. Graf, M. Parkinson, M. Wilhelm and M. Gaborieau, Detection and quantification of branching in polyacrylates by size-exclusion chromatography (SEC) and melt-state ${ }^{13} \mathrm{C}$ NMR spectroscopy, Polymer, 2009, 50(11), 2373-2383.

42 L. Voorhaar and R. Hoogenboom, Supramolecular polymer networks: hydrogels and bulk materials, Chem. Soc. Rev., 2016, 45, 4013-4031.

43 X. Wang, X. Guo, H. Wang and P. Guo, Effect of linearhyperbranched amphiphilic phosphate esters on collagen fibers, J. Agric. Food Chem., 2017, 65(1), 104-116.

44 P. Kucharczyk, J. Zednik, P. Humpolicek, Z. Capakova and V. Sedlarik, Versatile synthesis of comb-shaped poly(lactic acid) copolymers with poly(acrylic acid)-based backbones and carboxylic acid end groups, React. Funct. Polym., 2017, 111, 79-87.

45 W. Xu, P. A. Ledin, W. Shevchenko and W. Tsukruk, Architecture, assembly, and emerging applications of branched functional polyelectrolytes and poly(ionic liquid)s, ACS Appl. Mater. Interfaces, 2015, 7(23), 12570-12596.

46 J. Wang, W. Wang and A. Wang, Synthesis, characterization and swelling behaviors of hydroxyethyl cellulose- $g$-poly(acrylic acid)/attapulgite superabsorbent composite, Polym. Eng. Sci., 2010, 50(5), 1019-1027.

47 W. Wang, Y. Kang and A. Wang, One-step fabrication in aqueous solution of a granular alginate-based hydrogel for fast and efficient removal of heavy metal ions, J. Polym. Res., 2013, 20(3), 101-110.

48 J. Zhao, N. Dehbari, W. Han, L. Huang and Y. Tang, Electrospun multi-scale hybrid nanofiber/net with enhanced water swelling ability in rubber composites, Mater. Des., 2015, 86, 14-21.

49 S. Zu and B. Han, Aqueous dispersion of graphene sheets stabilized by pluronic copolymers: Formation of supramolecular hydrogel, J. Phys. Chem. C, 2009, 113(31), 13651-13657.

50 M. Zhong, Y. Liu, Z. Liu, F. Shi, L. Zhang, M. Zhu and X. Xie, Dually cross-linked single network poly(acrylic acid) hydrogels with superior mechanical properties and water absorbency, Soft Matter, 2016, 12(24), 5420-5428.

51 U. Gulyuz and O. Okay, Self-healing poly(acrylic acid) hydrogels with shape memory behavior of high mechanical strength, Macromolecules, 2014, 47(19), 6889-6899.

52 J. Yang, C. Gong, F. Shi and X. Xie, High strength of physical hydrogels based on poly(acrylic acid)-g-poly(ethylene glycol) methyl ether: role of chain architecture on hydrogel properties, J. Phys. Chem. B, 2012, 116(39), 12038-12047.

53 J. W. Lee, S. Y. Kim, S. S. Kim, Y. M. Lee, K. H. Lee and S. J. Kim, Synthesis and characteristics of interpenetrating polymer network hydrogel composed of chitosan and poly (acrylic acid), J. Appl. Polym. Sci., 1999, 73(1), 113-120.

54 S. Y. Kim, H. S. Shin, Y. M. Lee and C. N. Jeong, Properties of electroresponsive poly (vinyl alcohol)/poly (acrylic acid) IPN 
hydrogels under an electric stimulus, J. Appl. Polym. Sci., 1999, 73(9), 1675-1683.

55 J. Shen, B. Yan, T. Li, Y. Long, N. Li and M. Ye, Mechanical, thermal and swelling properties of poly (acrylic acid)-graphene oxide composite hydrogels, Soft Matter, 2012, 8(6), 1831-1836.

56 Y. Huang, J. Lu and C. Xiao, Thermal and mechanical properties of cationic guar gum/poly(acrylic acid) hydrogel membranes, Polym. Degrad. Stab., 2007, 92(6), 1072-1081.

57 J. Yang, C. Han, J. Duan, M. Ma, X. Zhang, F. Xu, R. Sun and $\mathrm{X}$. Xie, Studies on the properties and formation mechanism of flexible nanocomposite hydrogels from cellulose nanocrystals and poly(acrylic acid), J. Mater. Chem., 2012, 22(42), 22467-22480. 